\title{
Propagation of Lower-Order Modes in a Radially Anisotropic Cylindrical Waveguide with Liquid Crystal Cladding
}

\author{
Tien-Jung Chen and Shu-Hsia Chen
}

\begin{abstract}
The propagation behavior of the four lower-order modes, $\mathbf{H E}_{11}, \mathbf{T E}_{01}, \mathbf{T M}_{01}$, and $\mathbf{H E}_{21}$, in a radially anisotropic cylindrical waveguide with liquid crystal cladding is studied both theoretically and experimentally. The cylindrical waveguide is a doubly-clad fiber with an isotropic core and inner cladding and a radially anisotropic outer cladding made of nematic liquid crystal. Theoretically, the propagation and decay constants for the $\mathbf{T E}_{01}$ and $\mathbf{T} \mathbf{M}_{01}$ modes are obtained by solving the wave equations exactly, while those for the $\mathrm{HE}_{11}$ and $\mathrm{HE}_{21}$ modes are derived using perturbation techniques under the weakly guiding approximation. It is predicted that in such a structure the guided $\mathbf{T E}_{01}$ mode can be separated from the leaky $\mathbf{H E}_{11}, \mathbf{T M}_{01}$, and $\mathrm{HE}_{21}$ modes. The theoretical results show good agreement with the experimental observations for a $3 \mathrm{~cm}$ long fiber cell with a 5 $\mu \mathrm{m}$ inner cladding radius.
\end{abstract}

\section{INTRODUCTION}

$\mathbf{L}$ IQUID crystals possess high optical anisotropy and their macroscopic optical properties can easily be altered by an external electrical field. These distinctive characteristics make liquid crystals attractive for many applications. In the past decade, much research effort has been devoted to exploring the combined use of optical fibers with liquid crystals. In most studies liquid crystals are used as cladding. Examples include liquid-crystal-clad tapered fibers [1], liquid-crystaloverlay side-polished fiber [2]-[4], directional couplers with liquid crystal sandwiched between two side polished fibers [5], and coaxial fiber couplers embedded in liquid crystal [6]. These studies have demonstrated the feasibility of using optical fibers clad with liquid crystal to build switches, modulators, polarizers, and couplers. There are also some studies in which liquid crystals are used as the fiber core [7]-[10]. Among those experimental studies that have used liquid crystals as the fiber cladding [1]-[6], none have made liquid crystals with a radial molecular director configuration. It has been proposed [11] that by using liquid crystals with a radial configuration as the fiber cladding, one may be able to fabricate polarization-sensitive fiber devices. An analysis of single-cladding anisotropic fibers with cylindrical polar principal axes has also been developed [12]. In a recent letter [13], we described how the flexible alignment of liquid crystal molecules can be exploited to

Manuscript received December 29, 1994; revised March 28, 1995. This work was partially supported by the National Science Council of the ROC under contract no. NSC 83-0417-M009-023.

The authors are with the Institute of Electro-Optical Engineering, National Chiao Tung University, Hsinchu, Taiwan, R.O.C

IEEE Log Number 9413036

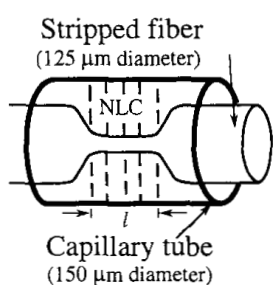

(a)

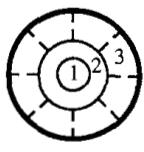

(b)
Fig. 1. (a) The geometry of the fiber cell: a section of length $l$ on the stripped fiber was etched and clad with liquid crystals. (b) A cross-sectional view of (a) showing the three-layer cylindrical structure: 1: core with radius $a, 2$ : inner cladding with radius $b, 3$ : NLC outer cladding.

prepare a fiber cell composed of cladding-removed fiber enclosed by radially aligned liquid crystals. Such a fiber cell can exhibit radially anisotropic optical properties which may have some applications. Experimentally, we have observed interesting mode selection behavior in such a radially anisotropic cylindrical waveguide [13]. In an isotropic cylindrical fiber, the $\mathrm{TE}_{01}$ and $\mathrm{TM}_{01}$ modes are difficult to separate because they have nearly the same propagation constants and cutoff characteristics. However, when a cylindrical fiber is clad with radially oriented liquid crystals, the $\mathrm{TE}_{01}$ and $\mathrm{TM}_{01}$ modes see different refractive index profiles because of their dissimilar polarization and thus exhibit different propagating properties. In this paper, we extend our previous investigations and present a theoretical formulation to describe the propagation behavior of the $\mathrm{HE}_{11}, \mathrm{TE}_{01}, \mathrm{TM}_{01}$, and $\mathrm{HE}_{21}$ modes - in the waveguide structure referred to above. We present a detailed analysis of the phenomena observed in our experiments.

The paper is organized as follows. The theoretical formulation is presented in Section II. The experimental setup is described in Section III. In Section IV we present theoretical and experimental results and compare the two sets of results. Conclusions are given in Section V.

\section{THEORY}

Fig. 1(a) shows a fiber cell of length $l$ composed of a cladding-removed fiber enclosed by radially oriented liquid crystals. The cross section of the fiber cell, shown in Fig. 1(b), indicates that the waveguide is a three-layer cylindrical structure with an isotropic core and inner cladding and a radially anisotropic outer cladding made of liquid crystal. The isotropic core and inner cladding have radii of $a$ and 


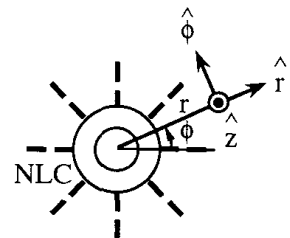

(a)

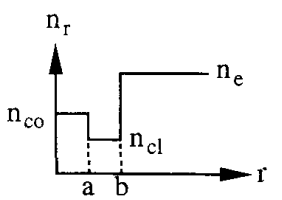

(b)

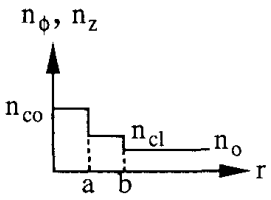

(c)

Fig. 2. Schematic drawings of (a) the cylindrical coordinate system and (b) the refractive index profile $n_{r}$, versus $r$, and (c) $n_{\phi}, n_{z}$ versus $r$.

$b$ and refractive indices $n_{c o}$ and $n_{c l}$, respectively. The liquid crystal outer cladding has extraordinary index $n_{e}$ and ordinary index $n_{o}$. In the derivation we use the cylindrical coordinates shown in Fig. 2(a). The principal axes of the outer cladding coincide with the axes of the cylindrical coordinates and the extraordinary principal axis is radially oriented, such that the refractive indices in outer cladding are $n_{r}=n_{e}$, $n_{\phi}=n_{z}=n_{o}$, and $n_{e}>n_{c o}>n_{c l}>n_{o}$. The whole profiles of the refractive index are shown in Fig. 2(b) and (c). In the following, we will consider the propagation of the four lower-order modes-the $\mathrm{HE}_{11}, \mathrm{TE}_{01}, \mathrm{TM}_{01}$, and $\mathrm{HE}_{21}$ modes-in this three-layer cylindrical waveguide with a radially anisotropic outer cladding.

Intuitively, the $\mathrm{HE}_{11}, \mathrm{HE}_{21}$, and $\mathrm{TM}_{01}$ modes have a nonzero $E_{r}$ component and can be expected to become leaky, because the $E_{r}$ component sees a high refractive index in the outer cladding, as shown in Fig. 2(b). On the other hand, the $\mathrm{TE}_{01}$ mode has only an $E_{\phi}$ component and should be guided, because it sees a low refractive index in the outer cladding, as shown in Fig. 2(c). To study the mode characteristics quantitatively, one must solve Maxwell's equations. For the $\mathrm{TE}_{01}$ and $\mathrm{TM}_{01}$ modes, the field components, which are independent of $\phi$, can be solved exactly, and the propagation constant $\beta$ can be obtained. However, it is difficult to obtain exact solutions for the $\mathrm{HE}_{11}$ and $\mathrm{HE}_{21}$ modes and thus we will use perturbation methods to estimate their propagation constants.

For an electromagnetic wave propagating along the $z$ direction with a frequency $\omega$ and a propagation constant $\beta$, the electric field vector can be written as $\overrightarrow{\mathbf{E}}=\overrightarrow{\mathbf{e}}(r, \phi) \exp [i(\omega t-$ $\beta z)$. The propagation constant $\beta$ is real for guided modes but is complex for leaky modes. From Maxwell's equations, one can obtain the following coupled equations for the transverse electrical field components $e_{r}$ and $e_{\phi}$ [11], [12]

$$
\begin{aligned}
{\left[\nabla_{t}^{2}+k^{2} n_{r}^{2}-\beta^{2}-\frac{1}{r^{2}}\right] e_{r}=} & \frac{2}{r^{2}} \frac{\partial e_{\phi}}{\partial \phi}+\left(1-\frac{n_{r}^{2}}{n_{z}^{2}}\right) \\
& \cdot \frac{\partial}{\partial r}\left[\frac{1}{r} \frac{\partial}{\partial}\left(r e_{r}\right)\right] \\
{\left[\nabla_{t}^{2}+k^{2} n_{\phi}^{2}-\beta^{2}-\frac{1}{r^{2}}\right] e_{\phi}=} & -\frac{2}{r^{2}} \frac{\partial e_{r}}{\partial \phi}+\left(1-\frac{n_{r}^{2}}{n_{z}^{2}}\right) \\
& \cdot \frac{1}{r^{2}} \frac{\partial}{\partial r}\left(r \frac{\partial e_{r}}{\partial \phi}\right)
\end{aligned}
$$

Here $\nabla_{t}^{2}=\partial^{2} / \partial r^{2}+(1 / r) \partial / \partial r+\left(1 / r^{2}\right) \partial^{2} / \partial \phi^{2}$ and $k$ is the free-space propagation constant.

\section{A. $T E_{01}$ Mode}

For the $\mathrm{TE}_{01}$ mode there is only one transverse electrical field component $e_{\phi}$ and it is independent of the coordinate $\phi$. That is, $e_{r}=0$ and $\partial e_{\phi} / \partial \phi=0$. In this case the equation for $e_{\phi}$ becomes

$$
\frac{\partial^{2} e_{\phi}}{\partial r^{2}}+\frac{1}{r} \frac{\partial e_{\phi}}{\partial r}+\left(k^{2} n_{\phi}^{2}-\beta^{2}-\frac{1}{r^{2}}\right) e_{\phi}=0
$$

Here, as shown in Fig. 2(c), $n_{\phi}$ is equal to $n_{c o}$ for $r \leq a, n_{c l}$ for $a \leq r \leq b$, and $n_{o}$ for $r \geq b$. Since $n_{c o}>n_{c l}>n_{o}$, (2) has a guided solution as follows:

$$
e_{\phi}(r)= \begin{cases}A_{\phi} J_{1}(u r) & r \leq a \\ B_{\phi} K_{1}(w r)+C_{\phi} I_{1}(w r) & a \leq r \leq b \\ D_{\phi} K_{1}(v r) & r \geq b\end{cases}
$$

where $A_{\phi}, B_{\phi}, C_{\phi}$, and $D_{\phi}$ are arbitrary constants to be determined and $u=\left(n_{c o}^{2} k^{2}-\beta^{2}\right)^{1 / 2}, w=\left(\beta^{2}-n_{c l}^{2} k^{2}\right)^{1 / 2}$, and $v=\left(\beta^{2}-n_{o}^{2} k^{2}\right)^{1 / 2}$. In addition to the electric field component $E_{\phi}=e_{\phi}(r) \exp [i(\omega t-\beta z)]$, there are magnetic field components $H_{r}=h_{r}(r) \exp [i(\omega t-\beta z)]$ and $H_{z}=$ $h_{z}(r) \exp [i(\omega t-\beta z)] . h_{r}$ and $h_{z}$ are related to $e_{\phi}$ by

$$
\begin{aligned}
& h_{r}=-\frac{\beta}{\omega \mu_{0}} e_{\phi}, \\
& h_{z}=\frac{i}{\omega \mu_{0}}\left(\frac{\partial e_{\phi}}{\partial r}+\frac{e_{\phi}}{r}\right) .
\end{aligned}
$$

Here $\mu_{0}$ is the permeability of free space. The continuity of the tangential field components $e_{\phi}$ and $h_{z}$ at interfaces $r=a$ and $b$ gives the following characteristic equation:

$$
\begin{aligned}
& {\left[\frac{u J_{0}(u a)}{J_{1}(u a)}+\frac{w K_{0}(w a)}{K_{1}(w a)}\right]} \\
& \cdot\left[\frac{w I_{0}(w b)}{I_{1}(w a)} \frac{K_{1}(v b)}{J_{1}(u a)}+\frac{I_{1}(w b)}{I_{1}(w a)} \frac{v K_{0}(v b)}{J_{1}(u a)}\right] \\
& \quad+\left[\frac{u J_{0}(u a)}{J_{1}(u a)}-\frac{w I_{0}(w a)}{I_{1}(w a)}\right] \\
& \cdot\left[\frac{w K_{0}(w b)}{K_{1}(w a)} \frac{K_{1}(v b)}{J_{1}(u a)}-\frac{K_{1}(w b)}{K_{1}(w a)} \frac{v K_{0}(v b)}{J_{1}(u a)}\right]=0
\end{aligned}
$$

where $J_{m}, I_{m}$, and $K_{m}$ are the Bessel and modified Bessel functions of the $m$ th order. The propagation constant $\beta$ for the $\mathrm{TE}_{01}$ mode is obtained by solving (6). 


\section{B. $T M_{01}$ Mode}

The derivation for the $\mathrm{TM}_{01}$ mode is similar to that for the $\mathrm{TE}_{01}$ mode. For the $\mathrm{TM}_{01}$ mode, the $e_{r}$ component is nonzero and is independent of the coordinate $\phi$. That is, $e_{\phi}=0$ and $\partial e_{r} / \partial \phi=0$. Other nonzero field components are the electric field component $E_{z}=e_{z}(r) \exp [i(\omega t-\beta z)]$ and the magnetic field component $H_{\phi}=h_{\phi}(r) \exp [i(\omega t-\beta z)]$. In this case, the equation for $e_{r}$ is

$$
\frac{\partial^{2} e_{r}}{\partial r^{2}}+\frac{1}{r} \frac{\partial e_{r}}{\partial_{r}}+\left[\left(k^{2} n_{r}^{2}-\beta^{2}\right) \eta-\frac{1}{r^{2}}\right] e_{r}=0
$$

and the other nonzero field components are related to $e_{T}$ by

$$
\begin{aligned}
& e_{z}=-\frac{i}{\beta \eta}\left(\frac{\partial e_{r}}{\partial r}+\frac{e_{r}}{r}\right) \\
& h_{\phi}=\frac{\omega \varepsilon_{0} n_{r}^{2}}{\beta} e_{r} .
\end{aligned}
$$

Here, as shown in Fig. 2(b), $n_{r}$ is equal to $n_{c o}$ for $r \leq a$, $n_{c l}$ for $a \leq r \leq b$, and $n_{e}$ for $r \geq b ; \eta=1$ in the isotropic region $(r \leq b)$ and $\eta=n_{o}^{2} / n_{e}^{2}$ in the liquid crystal anisotropic region $(r \geq b)$; and $\varepsilon_{0}$ is the permittivity of free space. Since $n_{e}>n_{c o}>n_{c l}$, (7) has the following leaky solution:

$$
e_{r}(r)= \begin{cases}A_{r} J_{1}(u r) & r \leq a \\ B_{r} K_{1}(w r)+C_{r} I_{1}(w r) & a \leq r \leq b \\ D_{r} H_{1}^{(2)}\left(v^{\prime} r\right) & r \geq b\end{cases}
$$

where $A_{r}, B_{r}, C_{r}$, and $D_{r}$ are undetermined coefficients and $u=\left(n_{c o}^{2} k^{2}-\beta^{2}\right)^{1 / 2}, w=\left(\beta^{2}-n_{c l}^{2} k^{2}\right)^{1 / 2}$, and $v^{\prime}=$ $\left[\left(n_{o} / n_{e}\right)^{2}\left(n_{e}^{2} k^{2}-\beta^{2}\right)\right]^{1 / 2}$. From the continuity of $h_{\phi}$ and $e_{z}$ at $r=a$ and $b$, we obtain

$$
\begin{aligned}
& {\left[\frac{u J_{0}(u a)}{n_{c o}^{2} J_{1}(u a)}+\frac{w K_{0}(w a)}{n_{c l}^{2} K_{1}(w a)}\right]} \\
& \cdot\left[\frac{w I_{0}(w b)}{n_{c l}^{2} I_{1}(w a)} \frac{H_{1}^{(2)}\left(v^{\prime} b\right)}{J_{1}(u a)}-\frac{I_{1}(w b)}{I_{1}(w a)} \frac{v^{\prime} H_{0}^{(2)}\left(v^{\prime} b\right)}{n_{o}^{2} J_{1}(u a)}\right] \\
& \quad+\left[\frac{u J_{0}(u a)}{n_{c o}^{2} J_{1}(u a)}-\frac{w I_{0}(w a)}{n_{c l}^{2} I_{1}(w a)}\right] \\
& \quad\left[\frac{w K_{0}(w b)}{n_{c l}^{2} K_{1}(w a)} \frac{H_{1}^{(2)}\left(v^{\prime} b\right)}{J_{1}(u a)}+\frac{K_{1}(w b)}{K_{1}(w a)} \frac{v^{\prime} H_{0}^{(2)}\left(v^{\prime} b\right)}{n_{o}^{2} J_{1}(u a)}\right]=0 .
\end{aligned}
$$

Here $H_{m}^{(2)}$ is the $m$ th order Hankel function of the second kind. The propagation constant $\beta$ for the $\mathrm{TM}_{01}$ mode is obtained by solving (11).

\section{C. $H E_{11}$ and $H E_{21}$ Modes}

The hybrid modes have both nonzero $e_{r}$ and $e_{\phi}$ transverse electric field components and can be expected to be leaky. To find the propagation constants, we have to solve the coupled equations (1a) and (1b) for $e_{r}$ and $e_{\phi}$. Since it is difficult to obtain exact solutions, we use the perturbation techniques developed in [12] to evaluate $\beta$ approximately.

We consider the anisotropic fiber cell to be a three-layer isotropic waveguide slightly perturbed by the anisotropic property in the outer cladding. The refractive index matrix of the anisotropic fiber can be expressed as

$$
\begin{aligned}
\overline{\mathbf{n}}^{2} & =\left[\begin{array}{ccc}
n_{r}^{2} & 0 & 0 \\
0 & n_{\phi}^{2} & 0 \\
0 & 0 & n_{z}^{2}
\end{array}\right] \\
& =n_{r}^{2}(\overline{\mathbf{I}}+\overline{\mathbf{D}}),
\end{aligned}
$$

where $\overline{\mathbf{I}}$ is a unit matrix and the matrix $\overline{\mathbf{D}}$ represents the anisotropic perturbation and is given by

$$
\overline{\mathbf{D}}=\left[\begin{array}{ccc}
0 & 0 & 0 \\
0 & \left(\frac{n_{\phi}^{2}}{n_{r}^{2}}\right) \delta_{r \phi} & 0 \\
0 & 0 & \left(\frac{n_{z}^{2}}{n_{r}^{2}}\right) \delta_{r z}
\end{array}\right] .
$$

Here $\delta_{r \phi}=\delta_{r z}=0$ in the isotropic region $(r \leq b)$ and $\delta_{r \phi}=\delta_{r z}=1-n_{e}^{2} / n_{o}^{2}$ in the liquid crystal anisotropic region $(r \geq b)$. If we introduce a scaling parameter $\lambda$ and let the perturbation be $\lambda \overline{\mathbf{D}}$, then $\overline{\mathbf{n}}^{2}=n_{r}^{2}(\overline{\mathbf{I}}+\lambda \overline{\mathbf{D}})$ and the square of propagation constant $\beta^{2}$ and the electric field $\overline{\mathbf{e}}$ can be expanded in power series of $\lambda$

$$
\begin{aligned}
\beta^{2} & =\beta_{0}^{2}+\lambda \beta_{1}^{2}+\lambda^{2} \beta_{2}^{2}+\cdots \\
\overrightarrow{\mathbf{e}} & =\overrightarrow{\mathbf{e}}_{0}+\lambda \overrightarrow{\mathbf{e}}_{1}+\lambda^{2} \overrightarrow{\mathbf{e}}_{2}+\cdots .
\end{aligned}
$$

Substituting the expressions for $\overline{\mathbf{n}}^{2}, \beta^{2}$, and $\overrightarrow{\mathbf{e}}$ into the (1) and equating the terms of the zero- and first-order in $\lambda$, we obtain

$$
\begin{aligned}
& {\left[\nabla_{t}^{2}+k^{2} n_{r}^{2}-\beta_{0}^{2}-\frac{1}{r^{2}}\right] e_{0 r}=\frac{2}{r^{2}} \frac{\partial e_{0 \phi}}{\partial \phi}} \\
& {\left[\nabla_{t}^{2}+k^{2} n_{r}^{2}-\beta_{0}^{2}-\frac{1}{r^{2}}\right] e_{0 \phi}=-\frac{2}{r^{2}} \frac{\partial e_{0 r}}{\partial \phi}}
\end{aligned}
$$

and

$$
\begin{aligned}
& {\left[\nabla_{t}^{2}+k^{2} n_{r}^{2}-\beta_{0}^{2}-\frac{1}{r^{2}}\right] e_{1 r}=} \\
& \quad \frac{2}{r^{2}} \frac{\partial e_{1 \phi}}{\partial \phi}+\beta_{1}^{2} e_{0 r}+\left(\frac{n_{z}^{2}}{n_{r}^{2}}\right) \delta_{r z} \frac{\partial}{\partial r}\left[\frac{1}{r} \frac{\partial}{\partial r}\left(r e_{0 r}\right)\right] \\
& {\left[\nabla_{t}^{2}+k^{2} n_{r}^{2}-\beta_{0}^{2}-\frac{1}{r^{2}}\right] e_{1 \phi}=} \\
& \quad-\frac{2}{r^{2}} \frac{\partial e_{1 r}}{\partial \phi}+\left(\beta_{1}^{2}-k^{2} n_{\phi}^{2} \delta_{r \phi}\right) e_{0 \phi} \\
& \quad+\left(\frac{n_{z}^{2}}{n_{r}^{2}}\right) \delta_{r z} \frac{1}{r^{2}} \frac{\partial}{\partial r}\left(r \frac{\partial e_{0 r}}{\partial \phi}\right) .
\end{aligned}
$$

In order to find the zero-order solution, we have to solve (16a) and (16b), which are exactly the propagation equations for an isotropic fiber with a refractive index profile $n_{r}(r)$ shown in Fig. 2(b). Under weakly guiding conditions, the zeroorder field components have the following solution form [14], [15]

$$
\begin{aligned}
e_{0 r}(r, \phi) & =f(r) \sin (n \phi), \\
e_{0 \phi}(r, \phi) & =f(r) \cos (n \phi) .
\end{aligned}
$$

Here $f(r)$ is given by

$$
f(r)= \begin{cases}A J_{n-1}(\tilde{u} r) & r \leq a \\ B K_{n-1}(\tilde{w} r)+C I_{n-1}(\tilde{w} r) & a \leq r \leq b \\ D H_{n-1}^{(2)}(\tilde{v} r) & r \geq b\end{cases}
$$


where $A, B, C$, and $D$ are undetermined coefficients, and $\tilde{u}=\left(n_{c o}^{2} k^{2}-\beta_{0}^{2}\right)^{1 / 2}, \tilde{w}=\left(\beta_{0}^{2}-n_{c l}^{2} k^{2}\right)^{1 / 2}$, and $\tilde{v}=$ $\left(n_{e}^{2} k^{2}-\beta_{0}^{2}\right)^{1 / 2}$. The $n$ refers to the first subscript of the $\mathrm{HE}_{n p}$ mode. By requiring $e_{0 \phi}$ and $\left(\partial e_{0 r} / \partial r+e_{0 r} / r\right)$ to be continuous at interfaces $r=a$ and $b$, which is deduced from the continuity of the $e_{0 \phi}$ and $e_{0 z}$ components, we obtain the following characteristic equation:

$$
\begin{aligned}
& {\left[\frac{\tilde{u} J_{n-2}(\tilde{u} a)}{J_{n-1}(\tilde{u} a)}+\frac{\tilde{w} K_{n-2}(\tilde{w} a)}{K_{n-1}(\tilde{w} a)}\right]} \\
& \cdot\left[\frac{\tilde{w} I_{n-2}(\tilde{w} b)}{I_{n-1}(\tilde{w} a)} \frac{H_{n-1}^{(2)}(\tilde{v} b)}{J_{n-1}(\tilde{u} a)}-\frac{I_{n-1}(\tilde{w} b)}{I_{n-1}(\tilde{w} a)} \frac{\tilde{v} H_{n-2}^{(2)}(\tilde{v} b)}{J_{n-1}(\tilde{u} a)}\right] \\
& \quad+\left[\frac{\tilde{u} J_{n-2}(\tilde{u} a)}{J_{n-1}(\tilde{u} a)}-\frac{\tilde{w} I_{n-2}(\tilde{w} a)}{I_{n-1}(\tilde{w} a)}\right] \\
& \quad \cdot\left[\frac{\tilde{w} K_{n-2}(\tilde{w} b)}{K_{n-1}(\tilde{w} a)} \frac{H_{n-1}^{(2)}(\tilde{v} b)}{J_{n-1}(\tilde{u} a)}+\frac{K_{n-1}(\tilde{w} b)}{K_{n-1}(\tilde{w} a)} \frac{\tilde{v} H_{n-2}^{(2)}(\tilde{v} b)}{J_{n-1}(\tilde{u} a)}\right] \\
& =0
\end{aligned}
$$

and the relations of the coefficients $A, B, C$, and $D$ are

$$
\begin{aligned}
& B=a\left[J_{n-1}(\tilde{u} a) \tilde{w} I_{n-2}(\tilde{w} a)-\tilde{u} J_{n-2}(\tilde{u} a) I_{n-1}(\tilde{w} a)\right] A \\
& C=a\left[J_{n-1}(\tilde{u} a) \tilde{w} K_{n-2}(\tilde{w} a)+\tilde{u} J_{n-2}(\tilde{u} a) K_{n-1}(\tilde{w} a)\right] A \\
& D=\frac{1}{H_{n-1}^{(2)}(\tilde{v} b)}\left[B K_{n-1}(\tilde{w} b)+C I_{n-1}(\tilde{w} b)\right] .
\end{aligned}
$$

From (20), the zero-order propagation constant $\beta_{0}$ can be evaluated and then the coefficients $A, B, C$, and $D$ can be determined from (21).

To find the first-order propagation constant $\beta_{1}$, we consider the integral of the expression $\left[e_{0 r} \times(17 \mathrm{a})-e_{1 r} \times(16 \mathrm{a})+\right.$ $\left.e_{0 \phi} \times(17 \mathrm{~b})-e_{1 \phi} \times(16 \mathrm{~b})\right]$ over the cross-sectional area of the anisotropic fiber (see Appendix). The first-order propagation constant $\beta_{1}$ obtained from this integration can be expressed analytically as

$$
\begin{aligned}
\beta_{1}^{2}= & \left(1-\frac{n_{o}^{2}}{n_{e}^{2}}\right)\left\{( k ^ { 2 } n _ { e } ^ { 2 } + \tilde { v } ^ { 2 } ) \left[H_{1}^{(2)^{2}}(\tilde{v} b)\right.\right. \\
& \left.\left.+H_{0}^{(2)^{2}}(\tilde{v} b)\right]-\frac{1}{b^{2}} H_{0}^{(2)^{2}}(\tilde{v} b)\right\} / \\
& 2\left[\frac{A^{2}}{D^{2}} \frac{a^{2}}{b^{2}}\left(1+\frac{\tilde{u}^{2}}{\tilde{w}^{2}}\right) J_{1}^{2}(\tilde{u} a)\right. \\
& \left.-\left(1+\frac{\tilde{v}^{2}}{\tilde{w}^{2}}\right) H_{1}^{(2)^{2}}(\tilde{v} b)\right]
\end{aligned}
$$

for the $\mathrm{HE}_{11}$ mode and

$$
\begin{aligned}
\beta_{1}^{2}= & \left(1-\frac{n_{o}^{2}}{n_{e}^{2}}\right)\left(( k ^ { 2 } n _ { e } ^ { 2 } + \tilde { v } ^ { 2 } ) \left\{H _ { 0 } ^ { ( 2 ) ^ { 2 } } ( \tilde { v } b ) \left[1-\frac{2}{\tilde{v} b}\right.\right.\right. \\
& \left.\left.\left.\cdot \frac{H_{1}^{(2)}(\tilde{v} b)}{H_{0}^{(2)}(\tilde{v} b)}\right]+H_{1}^{(2)^{2}}(\tilde{v} b)\right\}+\frac{2}{b^{2}} H_{0}^{(2)^{2}}(\tilde{v} b)\right) / \\
& 2\left\{\frac{A^{2}}{D^{2}} \frac{a^{2}}{b^{2}}\left(1+\frac{\tilde{u}^{2}}{\tilde{w}^{2}}\right) J_{0}^{2}(\tilde{u} a)\left[1-\frac{2}{\tilde{u} a} \frac{J_{1}(\tilde{u} a)}{J_{0}(\tilde{u} a)}\right]\right. \\
& \left.-\left(1+\frac{\tilde{v}^{2}}{\tilde{w}^{2}}\right) H_{0}^{(2)^{2}}(\tilde{v} b)\left[1-\frac{2}{\tilde{v} b} \frac{H_{1}^{(2)}(\tilde{v} b)}{H_{0}^{(2)}(\tilde{v} b)}\right]\right\}
\end{aligned}
$$

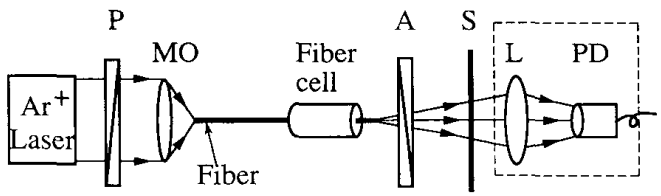

Fig. 3. Experimental setup: P: polarizer, MO: microscopic objective, A: analyzer, S: screen, L: collecting lens, PD: photodiode.

for the $\mathrm{HE}_{21}$ mode, where the constant $A^{2} / D^{2}$ is calculated using (21). Once $\beta_{0}$ is known, the first-order propagation constant $\beta_{1}$ can be obtained from (22) and (23). Finally, the propagation constant $\beta$ of the hybrid modes $\mathrm{HE}_{11}$ and $\mathrm{HE}_{21}$ can be estimated from $\beta^{2} \cong \beta_{0}^{2}+\beta_{1}^{2}$, in which $\lambda=1$ is set. The parameter $\lambda$ used in the previous perturbation procedure is for the purpose of tracing the development of the perturbed solutions from the unperturbed solutions.

\section{EXPERIMENTAL DESCRIPTION}

The optical fiber used was a single-mode fiber at the 633 $\mathrm{nm}$ wavelength. The core and cladding had diameters of 4 and $125 \mu \mathrm{m}$ and refractive indices of 1.462 and 1.458 , respectively. This fiber was commercially available step-index type (from Newport, Inc.) and at the $514.5 \mathrm{~nm}$ wavelength, the $\mathrm{HE}_{11}$, $\mathrm{TE}_{01}, \mathrm{TM}_{01}$, and $\mathrm{HE}_{21}$ modes are all above cut-off. The nematic liquid crystal used was mixture 14616, purchased from Merck. The ordinary index and extraordinary index were given as 1.457 and 1.5037 , respectively, at a wavelength of $509 \mathrm{~nm}$ and a temperature of $25^{\circ} \mathrm{C}$. The geometry of the fiber cell fabricated in our laboratory is shown in Fig. 1. One section of length $l=3 \mathrm{~cm}$ on the stripped fiber was etched using hydrofluoric acid $(48 \% \mathrm{HF})$ to remove parts of the original cladding. The cladding left had a diameter of $2 b=10 \mu \mathrm{m}$. Then the etched section was coated with DMOAP (N, Ndimethyl-N-octadecyl-3-aminopropyltrimethoxysilyl chloride) to achieve homeotropical alignment of the liquid-crystal directors on the boundary. The radial configuration of the liquid crystal was obtained by capillary action after the treated section of the fiber was inserted into a DMOAP coated capillary tube with an inner diameter of $150 \mu \mathrm{m}$ [16].

The experimental setup is shown in Fig. 3. After passing through the polarizer, an argon laser beam with a wavelength of $514.5 \mathrm{~nm}$ was focused into the fiber by a twenty times microscopic objective. The input power was $30 \mathrm{mw}$. After the output beam passed through the analyzer, the pattern of the beam was examined on the screen and the output power was measured by collecting the light to the photodiode.

\section{RESULTS AND DISCUSSION}

\section{A. Theoretical Results}

The theoretical formulas are given in (6), (11), and (20)-(23) in Section II. From these equations, numerical calculation of the propagation constant $\beta$ was carried out for the four lowerorder modes. For the transverse modes, i.e., the $\mathrm{TE}_{01}$ and $\mathrm{TM}_{01}$ modes, the evaluation is exact while for the hybrid mode case, i.e., the $\mathrm{HE}_{11}$ and $\mathrm{HE}_{21}$ modes, the calculation is to the first-order accuracy under weakly guiding conditions. 
The common parameters used were $n_{c o}=1.462, n_{c l}=1.458$, $n_{e}=1.5037, n_{o}=1.457$, and $a=2 \mu \mathrm{m}$, and the wavelength was $0.5145 \mu \mathrm{m}$. We define the mode index and the decay constant as $n_{e f f}=\beta_{r} / k$ and $\alpha=-2 \beta_{i}$, where $\beta_{r}$ and $\beta_{i}$ are the real and the imaginary parts of the propagation constant, respectively. The numerical results of the mode index $n_{\text {eff }}$ and the decay constant $\alpha$ versus the inner cladding radius $b$ are shown in Fig. 4(a) and (b). Fig. 4(a) indicates that the $\mathrm{HE}_{11}$ fundamental mode has the highest mode index. At a larger inner cladding radius $b$, the $\mathrm{TE}_{01}, \mathrm{TM}_{01}$, and $\mathrm{HE}_{21}$ modes are nearly degenerate. Their index values are very close to, but greater than, that of the inner cladding. However, when $b$ is gradually reduced, the mode indices of the $\mathrm{TE}_{01}, \mathrm{TM}_{01}$, and $\mathrm{HE}_{21}$ modes are split, such that for $b<4 \mu \mathrm{m}$, the index value of the $\mathrm{TE}_{01}$ mode remains greater than that of the inner cladding, whereas the index values of the $\mathrm{TM}_{01}$ and $\mathrm{HE}_{21}$ modes become less than that of the inner cladding. Fig. 4(b) shows the decay constants of the four modes: the $\mathrm{HE}_{11}, \mathrm{TM}_{01}$, and $\mathrm{HE}_{21}$ modes are leaky with a nonvanishing decay constant $\alpha$ and the $\mathrm{TE}_{01}$ mode is guided with a zero decay constant. This suggests that in this waveguide structure the $\mathrm{TE}_{01}$ mode can be separated from the $\mathrm{HE}_{11}, \mathrm{TM}_{01}$, and $\mathrm{HE}_{21}$ modes owing to their entirely different propagation behavior. In this respect, the inner cladding thickness plays an important role because the decay constant $\alpha$ for the leaky modes increases dramatically as the inner cladding radius $b$ is reduced. For $b<4 \mu \mathrm{m}$, the power decays of the $\mathrm{TM}_{01}$ and $\mathrm{HE}_{21}$ modes are due to the refractive light leakage, since their mode index values $n_{\text {eff }}$ are less than that of the inner cladding $n_{c l}$. On the other hand, for $b<4 \mu \mathrm{m}$, the power decay of the $\mathrm{HE}_{11}$ mode is due to the tunneling light leakage because $n_{\text {eff }}>n_{c l}$. When $b>4 \mu \mathrm{m}$, the leaky losses of all three modes are due to the tunneling effect. At $b=5 \mu \mathrm{m}$, the values of $\alpha$ are estimated to be $0.42,4.7$, and $5.75 \mathrm{~cm}^{-1}$ for the $\mathrm{HE}_{11}, \mathrm{TM}_{01}$, and $\mathrm{HE}_{21}$ modes, respectively. The decay constant of the $\mathrm{HE}_{11}$ mode is obviously smaller than those of the $\mathrm{TM}_{01}$ and $\mathrm{HE}_{21}$ modes. This is reasonable because the power of the $\mathrm{HE}_{11}$ mode is mostly concentrated in the core region and the tunneling loss through the evanescent field in the outer cladding is thus less than those of the $\mathrm{TM}_{01}$ and $\mathrm{HE}_{21}$ modes. To be more specific, a power transmittivity $T=e^{-\alpha l}$ is defined as the fractional power transmitted through the cell, where $l$ is the cell length. Corresponding to the experimental condition of $b=5 \mu \mathrm{m}$ and $l=3 \mathrm{~cm}$, the calculated transmittivity $T \approx$ $1,10^{-0.5}, 10^{-6}, 10^{-7.5}$ for the $\mathrm{TE}_{01}, \mathrm{HE}_{11}, \mathrm{TM}_{01}$, and $\mathrm{HE}_{21}$ modes, respectively. In this case the transmittivities of the $\mathrm{TM}_{01}$ and $\mathrm{HE}_{21}$ modes are small enough to be neglected.

\section{B. Experimental Results}

The fiber cell used in this study was designed to have a length of $3 \mathrm{~cm}$ and an inner cladding radius of $5 \mu \mathrm{m}$. An argon laser beam passed through a polarizer was coupled into the fiber cell and the output beam was examined with an analyzer and a screen. Fig. 5 shows the typical optical patterns observed on the screen when the analyzer was rotated. Part of the results appeared in our previous report [13] and are included here for completeness. Fig. 5(a) shows the optical pattern with the

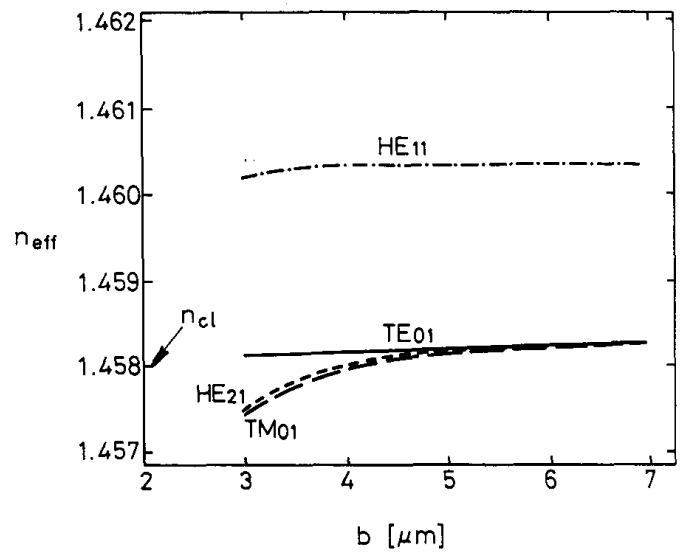

(a)

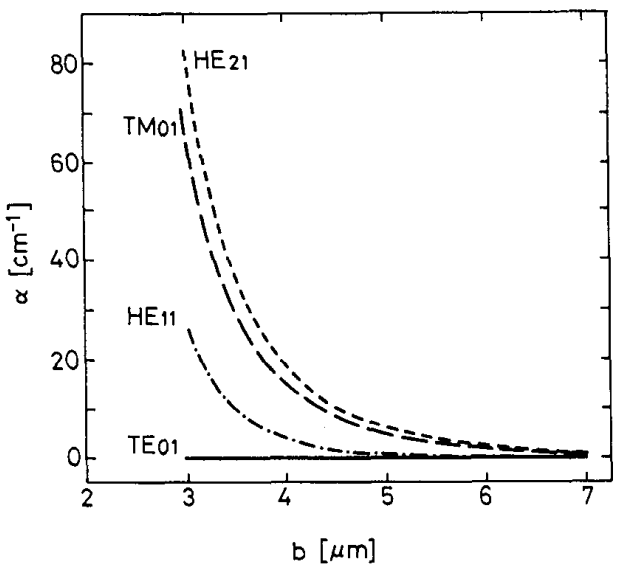

(b)

Fig. 4. The calculated results of (a) the mode index $n_{e f f}$ versus inner cladding radius $b$ and (b) the decay constant $\alpha$ versus inner cladding radius $b$.

analyzer parallel to the polarization of the input laser beam. With the analyzer perpendicular to the polarization of the input beam, the symmetric bisected pattern shown in Fig. 5(b) was obtained, where the null line is parallel to the direction of the analyzer. When the analyzer was rotated counterclockwise, the null line also rotated counterclockwise and an asymmetric pattern of two unequal parts, as shown in Fig. 5(c), was obtained. Further rotation of the analyzer caused one part of the asymmetric pattern to disappear completely at an angle $\delta_{0}$, as shown in Fig. 5(e). The value of $\delta_{0}$ depends on the input excitation condition. Fig. 5(d) and (f) show the asymmetric optical patterns obtained by rotating the analyzer clockwise.

To understand these results, let us now compare the patterns obtained experimentally with the optical patterns illustrated in Fig. 6. First, with a vertical analyzer, the $\mathrm{HE}_{11}$ mode is blocked out, and the existence of a null line coinciding with the direction of the analyzer indicates that the $\mathrm{TM}_{01}$ mode and one degenerate state (a) of the $\mathrm{HE}_{21}$ mode are absent. The corresponding experimental results are displayed in Fig. 5(b). Second, when the analyzer is rotated, the null line rotates in the same direction as the analyzer. This indicates that the other degenerate state (b) of the $\mathrm{HE}_{21}$ mode is absent. The 


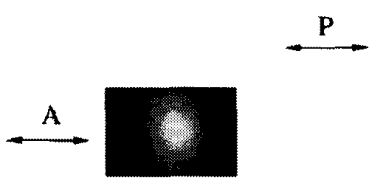

(a)

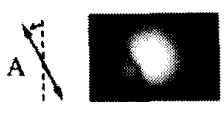

(c)

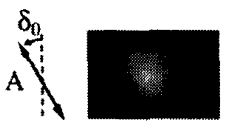

(e)

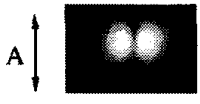

(b)

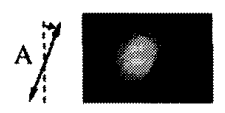

(d)

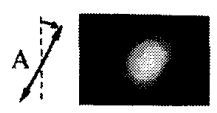

(f)

Fig. 5. Optical patterns of the output beam after passing through the analyzer. Pattern in (a) was under different exposure conditions. P: polarizer, A: analyzer, $\delta_{0}$ : the inclined angle of analyzer in (e).

\begin{tabular}{|l|lll}
\hline $\begin{array}{l}\text { Electric Field } \\
\text { Distribution }\end{array}$ & ME11 & TE01 \\
\hline $\begin{array}{l}\text { Optical Pattern } \\
\text { after Vertical } \\
\text { Analyzer }\end{array}$ & Empty \\
\hline $\begin{array}{l}\text { Optical Pattern } \\
\text { after Inclined } \\
\text { Analyzer }\end{array}$ & TM01
\end{tabular}

Fig. 6. Schematic drawing of the electric field vectors of the $\mathrm{TE}_{01}, \mathrm{HE}_{11}$, $\mathrm{TM}_{01}$, and $\mathrm{HE}_{21}$ modes before and after passing through the analyzer.

corresponding experimental results are shown in Fig. 5(c)-(f). Therefore, we conclude that the $\mathrm{TM}_{01}$ and $\mathrm{HE}_{21}$ modes have radiated out and only the $\mathrm{TE}_{01}$ and $\mathrm{HE}_{11}$ modes remain in the light beam coming out from the cell. The asymmetric patterns shown in Fig. 5(c)-(f) are precisely the results of the admixture of the $\mathrm{TE}_{01}$ and $\mathrm{HE}_{11}$ modes (see below). Our experimental observations are in agreement with the theoretical prediction that the transmittivities of the propagation modes $\mathrm{TM}_{01}$ and $\mathrm{HE}_{21}$ are negligibly small $\left(<10^{-6}\right)$ compared to those of the $\mathrm{TE}_{01}$ and $\mathrm{HE}_{11}$ modes $(0.3 \sim 1)$.

More details can be seen from Fig. 6. After passing through an inclined analyzer, a light beam composed of the $\mathrm{HE}_{11}$ and $\mathrm{TE}_{01}$ modes will produce an asymmetric pattern. This is because the symmetric optical pattern obtained from the $\mathrm{TE}_{01}$ mode is partially enhanced and partially weakened by the $\mathrm{HE}_{11}$ mode. As the analyzer is rotated, for the $\mathrm{TE}_{01}$ mode the maximum value of the electric field remains the same, whereas for the $\mathrm{HE}_{11}$ mode it varies. When the analyzer is rotated, the maximum electric field of the $\mathrm{TE}_{01}$ mode occurs, with opposite polarization, at two positions. We follow one of these two positions and determine the inclined angle of analyzer at which the electric field of the $\mathrm{HE}_{11}$ mode just cancels that of the $\mathrm{TE}_{01}$ mode. This angle $\delta_{0}$ is related to the power ratio of the $\mathrm{TE}_{01}$ mode to the $\mathrm{HE}_{11}$ mode before passing through the analyzer. Denoting this ratio by $P_{r}$, we find $P_{r}=1.25 \sin ^{2} \delta_{0}$. The angle $\delta_{0}$ can be measured experimentally as shown in Fig.

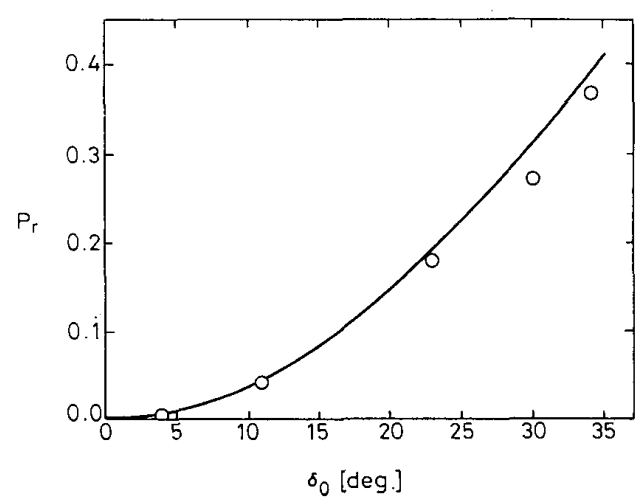

Fig. 7. Experimental results for the power ratio $P_{r}$ versus the angle $\delta_{0}$; the solid line is plotted as a function $1.25 \sin ^{2} \delta_{0}$.

5(e). The individual powers of the $\mathrm{TE}_{01}$ and $\mathrm{HE}_{11}$ modes can be obtained by measuring the light beam through the analyzer in the cases shown in Fig. 5(a) and (b), respectively. This is because the power measured in the case shown in Fig. 5(a) is the sum of $(1 / 2) \mathrm{TE}_{01}$ and $\mathrm{HE}_{11}$, and that in the case shown in Fig. 5(b) is (1/2) $\mathrm{TE}_{01}$. In this way the power ratio of the $\mathrm{TE}_{01}$ mode to the $\mathrm{HE}_{11}$ mode can be determined. The ratio $P_{r}$ varies with the conditions under which the light beam is coupled into the fiber. As shown in Fig. 7, the measured $P_{r}$ versus the angle $\delta_{0}$ confirms the relation derived. This indicates that the angle $\delta_{0}$ observed on the optical pattern can be used as an index for the power ratio of the $\mathrm{TE}_{01}$ mode to the $\mathrm{HE}_{11}$ mode in the output beam from the cell.

In our experiment $P_{r}$ was less than one. This implies that the $\mathrm{HE}_{11}$ mode has a greater power than the $\mathrm{TE}_{01}$ mode in the output beam, even though the theoretical calculations predict that the intrinsic characteristics of the fiber cell yield a larger transmittivity for the guided $\mathrm{TE}_{01}$ mode $(\sim 1)$ than for the leaky $\mathrm{HE}_{11}$ mode $(\sim 0.3)$. Because the field distribution of the $\mathrm{HE}_{11}$ mode is more matched to the incident laser beam, which has a gaussian profile and a linear polarization, the $\mathrm{HE}_{11}$ mode is excited more efficiently at the input end of the fiber. Therefore, even though it has a lower transmittivity, the $\mathrm{HE}_{11}$ mode still dominates in the output beam.

\section{CONCLUSION}

We have studied the propagation properties of the $\mathrm{HE}_{11}$, $\mathrm{TE}_{01}, \mathrm{TM}_{01}$, and $\mathrm{HE}_{21}$ modes in a radially anisotropic cylindrical waveguide with liquid crystal outer cladding. For such a waveguide structure, the $\mathrm{TE}_{01}$ mode is guided while the $\mathrm{HE}_{11}$, $\mathrm{TM}_{01}$, and $\mathrm{HE}_{21}$ modes are leaky. Formulas for calculating the decay constants of these modes have been presented. The calculated results indicate that the decay constants of the leaky $\mathrm{HE}_{11}, \mathrm{TM}_{01}$, and $\mathrm{HE}_{21}$ modes are significantly influenced by the inner cladding radius $b$. As the inner cladding radius $b$ is reduced, the decay constants increase dramatically. For smaller $b(<4 \mu \mathrm{m})$, the propagating losses of the $\mathrm{TM}_{01}$ and $\mathrm{HE}_{21}$ modes are due to the refractive light leakage, while that of the $\mathrm{HE}_{11}$ mode is due to the tunneling light leakage. For larger $b(>4 \mu \mathrm{m})$, the losses are due to light tunneling for all leaky modes. At $b=5 \mu \mathrm{m}$, the decay constant of the $\mathrm{HE}_{11}$ 
mode is shown to be far smaller than those of the $\mathrm{TM}_{01}$ and $\mathrm{HE}_{21}$ modes. This is because most of the power of the $\mathrm{HE}_{11}$ mode is concentrated in the core and thus the tunneling loss contributed from the evanescent field at the outer cladding is less than those of the other two leaky modes, $\mathrm{TM}_{01}$ and $\mathrm{HE}_{21}$. For a 3-cm-long fiber cell with an inner cladding radius $b=5$ $\mu \mathrm{m}$, the theoretical results show that the transmittivities of the $\mathrm{TM}_{01}$ and $\mathrm{HE}_{21}$ modes are negligibly small compared with those of the $\mathrm{TE}_{01}$ and $\mathrm{HE}_{11}$ modes. This agrees well with our experimental observations. In the experiment, it was found that because the field distribution of the $\mathrm{HE}_{11}$ mode is more matched to the profile of the input laser beam, even though the $\mathrm{HE}_{11}$ mode is leaky when propagating through the waveguide, the output beam still has a significant $\mathrm{HE}_{11}$ component in addition to the guided $\mathrm{TE}_{01}$ mode.

The modal filtering in a radially anisotropic cylindrical optical fiber has been demonstrated in this study. Such a fiber in which two spatial modes, $\mathrm{TE}_{01}$ and $\mathrm{HE}_{11}$, are retained may be exploited to develop new dual-mode fiber-optic devices with a possible application in interferometric sensing. A further exploration is in progress.

\section{APPENDIX}

The First Order Propagation Constant $\beta_{1}$ of $\mathrm{HE}_{11}$ and $\mathrm{HE}_{21}$ Modes

We start from the integral of the expression $\left[e_{0 r} \times\right.$ $\left.(17 \mathrm{a})-\mathrm{e}_{1 r} \times(16 \mathrm{a})+\mathrm{e}_{0 \phi} \times(17 \mathrm{~b})-\mathrm{e}_{1 \phi} \times(16 \mathrm{~b})\right]$ over the cross-sectional area of the anisotropic fiber

$$
\begin{aligned}
& \int_{A_{\infty}}\left(e_{0 r} \nabla_{t}^{2} e_{1 r}-e_{1 r} \nabla_{t}^{2} e_{0 r}+e_{0 \phi} \nabla_{t}^{2} e_{1 \phi}-e_{1 \phi} \nabla_{t}^{2} e_{0 \phi}\right) d s= \\
& \int_{A_{\infty}} \frac{2}{r^{2}}\left(e_{0 r} \frac{\partial e_{1 \phi}}{\partial \phi}-e_{1 r} \frac{\partial e_{0 \phi}}{\partial \phi}-e_{0 \phi} \frac{\partial e_{1 r}}{\partial \phi}+e_{1 \phi} \frac{\partial e_{0 r}}{\partial \phi}\right) d s \\
& +\int_{A_{\infty}}\left\{\beta_{1}^{2} e_{0 r}^{2}+\left(\beta_{1}^{2}-k^{2} n_{\phi}^{2} \delta_{r \phi}\right) e_{0 \phi}^{2}+\left(\frac{n_{z}^{2}}{n_{r}^{2}}\right) \delta_{r z}\right. \\
& \left.\cdot\left[e_{0 r} \frac{\partial}{\partial r}\left(\frac{1}{r} \frac{\partial}{\partial r} r e_{0 r}\right)+e_{0 \phi} \frac{1}{r^{2}} \frac{\partial}{\partial r}\left(r \frac{\partial e_{0 r}}{\partial \phi}\right)\right]\right\} d s .
\end{aligned}
$$

The area integral on the left side of (Al) can be transformed to the following line integrals by Green's theorem:

$$
\begin{aligned}
& \lim _{\varepsilon \rightarrow 0} \oint_{c}\left[\left(e_{0 r} \frac{\partial e_{1 r}}{\partial r}-e_{1 r} \frac{\partial e_{0 r}}{\partial r}\right)\right. \\
& \left.+\left(e_{0 \phi} \frac{\partial e_{1 \phi}}{\partial r}-e_{1 \phi} \frac{\partial e_{0 \phi}}{\partial r}\right)\right]_{r=a+\varepsilon}^{r=a-\varepsilon} d l \\
& \quad+\lim _{\varepsilon^{\prime} \rightarrow 0} \oint_{c^{\prime}}\left[\left(e_{0 r} \frac{\partial e_{1 r}}{\partial r}-e_{1 r} \frac{\partial e_{0 r}}{\partial r}\right)\right. \\
& \left.+\left(e_{0 \phi} \frac{\partial e_{1 \phi}}{\partial r}-e_{1 \phi} \frac{\partial e_{0 \phi}}{\partial r}\right)\right]_{r=b+\varepsilon^{\prime}}^{r=b-\varepsilon^{\prime}} d l^{\prime} .
\end{aligned}
$$

In the weakly guiding case, the components $e_{r}$ and $e_{\phi}$ and their derivatives $\partial e_{r} / \partial r$ and $\partial e_{\phi} / \partial r$ are continuous at boundaries $r=a$ and $b$, and the above line integrals vanish. The first term on the right side of (A1) can be proved to vanish because the field components $e_{r}$ and $e_{\phi}$ are single-valued functions of coordinate $\phi$. Thus (A1) can be reduced to contain only the last integral term, i.e.

$$
\begin{gathered}
\int_{A_{\infty}}\left\{\beta_{1}^{2} e_{0 r}^{2}+\left(\beta_{1}^{2}-k^{2} n_{\phi}^{2} \delta_{r \phi}\right) e_{0 \phi}^{2}\right. \\
+\left(\frac{n_{z}^{2}}{n_{r}^{2}}\right) \delta_{r z}\left[e_{0 r} \frac{\partial}{\partial r}\left(\frac{1}{r} \frac{\partial}{\partial r} r e_{0 r}\right)\right. \\
\left.\left.+e_{0 \phi} \frac{1}{r^{2}} \frac{\partial}{\partial r}\left(r \frac{\partial e_{0 r}}{\partial \phi}\right)\right]\right\} d s=0 .
\end{gathered}
$$

The first-order propagation constant can be obtained as follows:

$$
\begin{aligned}
\beta_{1}^{2}= & \int_{A_{\infty}}\left\{\delta_{r \phi} k^{2} n_{\phi}^{2} e_{0 \phi}^{2}-\delta_{r z}\left(\frac{n_{z}^{2}}{n_{r}^{2}}\right)\right. \\
& \left.\cdot\left[e_{0 r} \frac{\partial}{\partial r}\left(\frac{1}{r} \frac{\partial}{\partial r} r e_{0 r}\right)+e_{0 \phi} \frac{1}{r^{2}} \frac{\partial}{\partial_{r}}\left(r \frac{\partial e_{0 r}}{\partial \phi}\right)\right]\right\} \\
& \cdot d s / \int_{A_{\infty}}\left(e_{0 r}^{2}+e_{0 \phi}^{2}\right) d s
\end{aligned}
$$

After the conditions of $\delta_{r \phi}=\delta_{r z}=0$ for $r \leq b$ and $\delta_{r \phi}=\delta_{r z}=1-n_{e}^{2} / n_{o}^{2}$ for $r \geq b$ are considered in the integral of (A4), in which $e_{0 r}$ and $e_{0 \phi}$ are given by (18) and (19), $\beta_{1}^{2}$ can be analytically expressed as (22) and (23), shown on page 1701 .

\section{ACKNOWLEDGMENT}

The authors would like to thank Prof. Y. Lai for useful discussions.

\section{REFERENCES}

[1] C. Veilleux, J. Lapierre, and J. Bures, "Liquid-crystal-clad tapered fibers," Opt. Lett., vol. 11, pp. 733-735, 1986.

[2] K. Liu, W. V. Sorin, and H. J. Shaw, "Single-mode-fiber evanescent polarizer/amplitude modulator using liquid crystals," Opt. Lett., vol. 11, pp. 180-182, 1986

[3] R. Kashyap, C. S. Winter, and B. K. Nayar, "Polarization-desensitized liquid-crystal overlay optical-fiber modulator," Opt. Lett., vol. 13, pp. $401-403,1988$.

[4] Z. K. Ioannidis, I. P. Giles, and C. Bowry, "All-fiber optic intensity modulators using liquid crystals," Appl. Opt., vol. 30, pp. 328-333, 1991.

[5] E. S. Goldburt and P. St. Russell, "Electro-optical response of a liquidcrystalline fiber coupler," Appl. Phys. Lett., vol. 48, pp. 10-12, 1986.

[6] V. I. Busurin, M. Green, J. R. Cozens, and K. D. Leaver, "Switchable coaxial optical coupler using a liquid crystal mixture," Appl. Phys. Lett., vol. 42 , pp. 322-324, 1983.

[7] M. Green and S. J. Madder, "Low loss nematic liquid-crystal cored fiber waveguides," Appl. Opt., vol. 28, pp. 5202-5203, 1989.

[8] S. K. Lo, L. M. Galarneau, D. J. Rogers, and S. R. Flow, "Smectic liquid crystal waveguides with cylindrical geometry," Mol. Cryst. Liq. Cryst., vol. 201, pp. 137-145, 1991.

[9] H. Lin, P. Palffy-Muhoray, and M. A. Lee, "Liquid crystalline cores for optical fibers," Mol. Cryst. Liq. Cryst., vol. 204, pp. 189-200, 1991.

[10] I. C. Khoo, H. Li, P. G. LoPresti, and Y. Liang, "Observation of optical limiting and backscattering of nanosecond laser pulses in liquid-crystal fibers," Opt. Lett., vol. 19, pp. 530-532, 1994.

[11] R. J. Black, C. Veilleux, J. Bures, and J. Lapierre, "Radially anisotropic lightguide mode selector," Electron. Lett., vol. 21, pp. 987-989, 1985.

[12] Y. Chen, "Anisotropic fiber with cylindrical polar axes," Appl. Phys. B, vol. 42 , pp. 1-3, 1987.

[13] S.-H. Chen and T.-J. Chen, "Observation of mode selection in a radially anisotropic cylindrical waveguide with liquid-crystal cladding," Appl. Phys. Lett, vol 64, pp. 1893-1895, 1994.

[14] S. Kawakami and S. Nishida, "Perturbation theory of a doubly clad optical fiber with a low-index inner cladding," IEEE J. Quantum Electron., vol. QE-11, pp. 130-138, 1975.

[15] M. Monerie, "Propagation in doubly clad single-mode fibers," IEEE $J$. Quantum Electron., vol. QE-18, pp. 535-542, 1982. 
[16] P. Palffy-Muhoray, A. Sparavigna, and A. Strigazzi, "Saddle-splay and mechanical instability in nematics confined to a cylindrical annular geometry," Liq. Cryst., vol. 14, pp. 1143-1151, 1993.

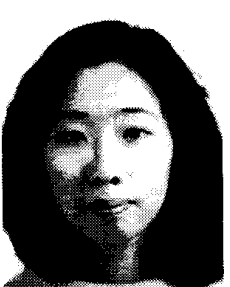

Tien-Jung Chen was born in Taipei, Taiwan, in 1963. She received the B.S. degree in electrical engineering from National Cheng Kung University, Tainan, Taiwan, in 1985 and the M.S. degree in electro-optical engineering from National Chiao Tung University, Hsinchu, Taiwan, in 1988.

She then joined the Electro-Optics \& Peripherals Development Center, Industrial Technology Research Institute (ITRI), Hsinchu, Taiwan, where she was engaged in the research on integrated optics. In 1989, she entered the Ph.D. program in the Institute of Electro-Optical Engineering at National Chiao Tung University. Her current studies have been on anisotropic liquid crystal waveguides.

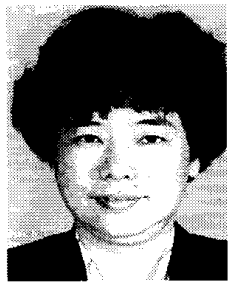

Shu-Hsia Chen received the B.S. degree in physics from National Taiwan Normal University, Taiwan, ROC, in 1968 and the M.S. degrees respectively in physics from North Carolina State University, Raleigh, NC, in 1970 and in Bionucleonics from Purdue University, West Lafayette, IN, in 1973.

Since 1974 , she has been on the faculty of $\mathrm{Na}$ tional Chiao Tung University, Taiwan, ROC, where she is currently a Professor of Electro-Optical Engineering. She served as Director of the Institute of Electro-Optical Engineering from 1990-1992. She spent the academic year 1984 at Lawrence Berkeley Laboratory, University of California, Berkeley, CA, as a guest researcher. During 1989-1990, she spent six months at the Department of Electrical Engineering. University of Maryland, College Park, MD, as a Visiting Scholar. Her current research interests focus on nonlinear optics of liquid crystals, closed cylindrical liquid crystal (CCLC), Polymer-dispersed liquid crystal (PDLC), and liquid crystal display (LCD). 